\title{
Adhesion of Gold Nanoparticles on an Electrochemically Pretreated Glassy Carbon Electrode
}

\author{
Hyosul SHIN and Chan KANG ${ }^{\dagger}$ \\ Department of Chemistry, Chonbuk National University, Chonju 561-756, The Republic of Korea
}

\begin{abstract}
This study describes a simple new method for the adsorption of gold nanoparticles on a glassy carbon (GC) electrode. By electrochemically oxidizing the GC electrode and immersing it into a gold colloidal solution, one could induce the adhesion of the gold nanoparticles. The image of the field emission scanning electron microscopy confirmed that the nanoparticles were evenly distributed over the surface. A 2-aminoethanethiol self-assembled monolayer was formed on the surfaces of the adsorbed gold nanoparticles and a reductive desorption wave was observed during the reduction of the adsorbed 2-aminoethanethiol.
\end{abstract}

(Received March 20, 2003; Accepted October 3, 2003)

\section{Introduction}

Studies using gold nanoparticles are containing the areas of catalysis and fabrications of nano-scale structures. ${ }^{1-5}$ In the search for catalysts in electrochemistry, electrode reactions or electrocatalytic reactions were carried out for the gold nanoparticles. ${ }^{2,6,7}$ The nanometer-sized monolayer-protected gold clusters behaved as soluble nanoelectrodes with multielectron transfer and electrical double layer charging properties. ${ }^{2}$ The electrode reactions of 1,1-dinitrocyclohexane, dioxygen, and carbon monoxide were catalyzed and some different catalytic activities were found in comparison to those of the bulk electrodes., ${ }^{2,6}$ The adsorption of the gold nanoparticles on electrode surfaces seems to be an interesting issue in the utilization for the electrode reactions. Reports of attachments of the gold nanoparticles on a glassy carbon (GC) electrode, which is widely used, are relatively rare. Their attachment to a mercaptobenzene film on a GC electrode was reported ${ }^{8}$ and another gold nanoparticle thin film was prepared by dissolving decanethiolate-encapsulated gold nanoparticles and 1,9-nonanedithiol in an organic solvent. ${ }^{9.10}$ In the latter process, as the exchange reaction proceeded, a thin film was deposited. Elsewhere, the gold nanocrystals were directly electrodeposited, and their shapes and sizes were controlled by applying potential and by time. ${ }^{11}$

In the present study, we report another simple method to adsorb the gold nanoparticles on a GC electrode. By electrochemically oxidizing the GC electrode and immersing it in a gold colloidal solution, one could induce the adsorption of the gold nanoparticles. The modified GC electrode showed a new interesting surface property giving a way to adsorb the nanoparticles on the GC electrode surface. Adsorptions of some chemical compounds on the GC surface after the electrochemical oxidation treatment were reported. ${ }^{12}$ Aromatic compounds, alkali metal cations, and halide anions were adsorbed on the electro-oxidized surface. ${ }^{13-15}$ Adsorbed gold

†To whom correspondence should be addressed.

E-mail: chankang@moak.chonbuk.ac.kr nanoparticles were characterized by field emission scanning electron microscopy and cyclic voltammetry. The interaction properties are discussed.

\section{Experimental}

\section{Reagents}

Commercial reagent grade chemicals were used as received. The deionized water was further purified by passage through a purification train (Human power $\mathrm{II}^{+}$, Human Co.) and used for solution preparations. A commercially available GC electrode (Kosentech Co.) was used as a working electrode. The gold colloidal particles were synthesized as reported. ${ }^{16}$ A solution containing $274 \mathrm{mg} \mathrm{HAuCl}_{4} \cdot 3 \mathrm{H}_{2} \mathrm{O}$ in $500 \mathrm{~mL}$ of water was boiled and $50 \mathrm{mg}$ of $1.0 \%$ sodium citrate solution was added. The solution was then boiled for $1 \mathrm{~h}$.

\section{Apparatus and procedure}

Electrochemical measurements were conducted in a twocompartment cell closed with a Teflon cap through which electrodes and a gas bubbling system were fitted. A GC working electrode (area, $0.071 \mathrm{~cm}^{2}$ ) and a platinum auxiliary electrode were in one compartment, separated by fritted glass from the other compartment where a reference electrode was held. A CH Instruments electrochemical analyzer (Model 630A) controlled through a personal computer was employed for the electrochemical measurements. Field emission scanning electron microscopy (FE-SEM) images were obtained with a Hitachi Model S-4700 instrument provided by the Korea Basic Science Institute.

For the electrochemical oxidation of the GC electrode, the potential was initially scanned to $2.0 \mathrm{~V}$ (vs. $\mathrm{Ag} / \mathrm{AgCl}$ ), held for $6 \mathrm{~min}$, and reversed to $-0.5 \mathrm{~V}$ in a $0.10 \mathrm{M} \mathrm{H}_{2} \mathrm{SO}_{4}$ (unless otherwise specified). ${ }^{17}$ Repeated scans between $0.5 \mathrm{~V}$ and -0.5 $\mathrm{V}$ after the oxidation treatment gave stable background currents. The treated electrode was immersed for $40 \mathrm{~min}$ in the gold colloidal solution to adsorb the gold nanoparticles. The immersion time was decided based on the reduction wave of the adsorbed thiol monolayer (vide infra). A freshly prepared 
electrode was used for each measurement. The electrode surface was made clean by grinding with 600 , and then with 1200 grit $\mathrm{SiC}$ paper, and the surface was finally polished with $0.3 \mu \mathrm{m}$ alumina powder. The polished electrode was sonicated for about $30 \mathrm{~s}$ in purified water before use. After the electrochemical oxidation of the electrode surface, this cleaning treatment produced a fresh surface, giving a flat voltammogram with low background currents.

Potentials were read and written with respect to the $\mathrm{Ag} / \mathrm{AgCl}$ $(3 \mathrm{M} \mathrm{NaCl})$ reference electrode with a potential of $0.22 \mathrm{~V} v s$. NHE. The supporting electrolyte was either a $0.1 \mathrm{M} \mathrm{H}_{2} \mathrm{SO}_{4}$ or $0.10 \mathrm{M}$ phosphate buffer ( $\mathrm{pH}$ 6.0). All experiments were carried out at the ambient temperature $\left(22 \pm 1^{\circ} \mathrm{C}\right)$.

\section{Results and Discussion}

The FE-SEM image of the gold nanoparticles adsorbed on the GC electrode

The adsorbed nanoparticles were visually observed using FESEM analysis. Figure 1 is a representative FE-SEM image showing round-shaped bright spots, which can be assigned to the gold nanoparticles adsorbed on the GC electrode with the dark background image. No such spots were found in another image with the identically pretreated electrode without immersing in the gold colloidal solution. The particle sizes of $20-30 \mathrm{~nm}$ were estimated. No aggregation of the particles on the surface occurred and the particles were evenly distributed. The number density of $1.2( \pm 0.3) \times 10^{10} \mathrm{~cm}^{-2}$ from the obtained image was as high as that of the electrodeposited nanocrystals on the GC electrode. ${ }^{11}$ For the controlled experiment, the GC electrode polished with $0.3 \mu \mathrm{m}$ alumina powder, which showed a mirror-like surface, was also immersed in the gold colloidal solution and another FE-SEM image was obtained. No adsorbed particles were shown, which indicated that the electrochemical oxidation treatment induced the adhesion of the gold nanoparticles on the surface.

\section{The adsorption of 2-aminoethanethiol on the gold nanoparticles} adsorbed on the GC electrode

Figure $2 \mathrm{~A}-\mathrm{a}$ is a cyclic voltammogram in a $0.1 \mathrm{M}$ phosphate buffer ( $\mathrm{pH}$ 6.0) with a GC electrode polished with $0.3 \mu \mathrm{m}$ alumina powder. The flat background voltammogram shows relatively low currents in the potential range. The voltammogram of Fig. 2A-b was obtained in the same supporting electrolyte solution with the GC electrode after the electrochemical oxidation treatment, as explained in the experimental section. The voltammogram showed greatly enhanced background currents in comparison to those in Fig. 2A-a. The enhancement of the background currents was ascribed to the double layer charging with the increased surface roughness, as well as to the faradaic reactions of some electroactive surface functional groups produced after the electrochemical pretreatment. ${ }^{14}$ The electro-oxidized GC electrode was immersed in the solution containing colloidal particles and then was transferred to the pure supporting electrolyte to obtain the voltammogram of Fig. 2A-c. At potentials more negative than $-0.9 \mathrm{~V}$, sharp tailing currents were measured. Similar currents were observed with a commonly used gold electrode. ${ }^{18}$ The tailing currents are thought to be due to the hydrogen evolution reaction in the absence of $\mathrm{O}_{2}$ at the adsorbed gold nanoparticles on the GC electrode surface.

We tried to form a self-assembled monolayer on the gold nanoparticles adsorbed. The formation of the self-assembled

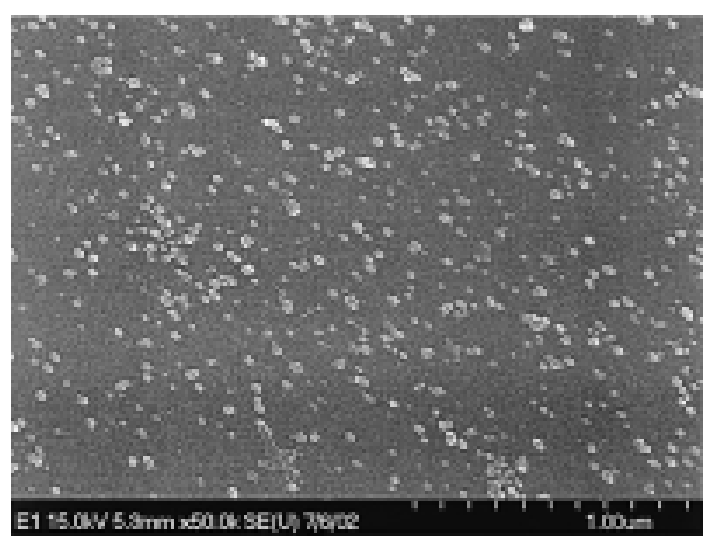

Fig. 1 A representative FE-SEM image of gold colloidal particles adsorbed on the electrochemically oxidized GC electrode. The 15.0 $\mathrm{kV}$ accelerating voltage was used and Pt was coated.

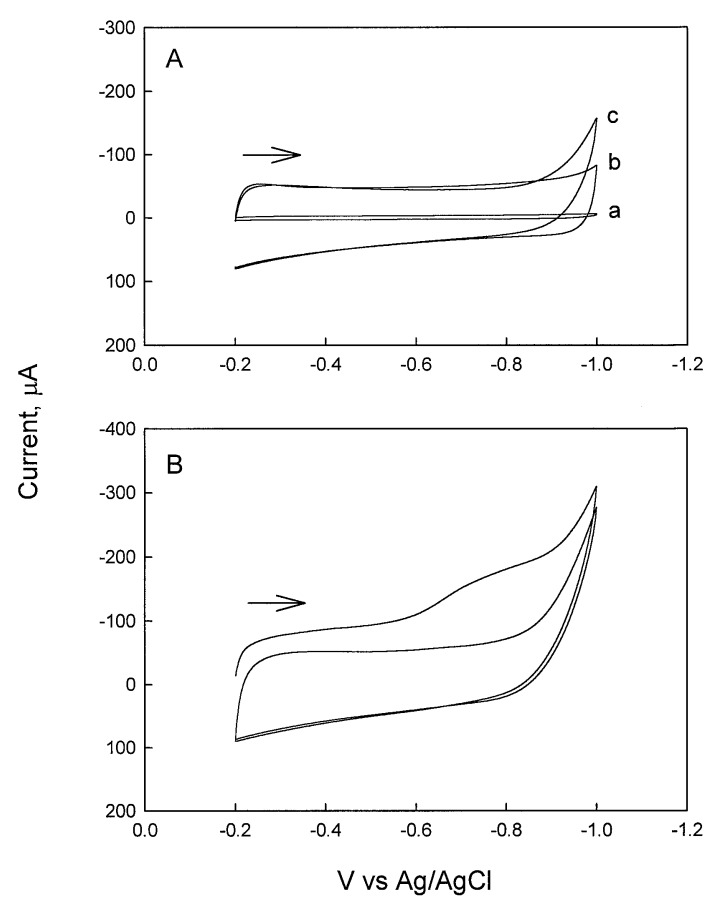

Fig. 2 (A) Cyclic voltammograms in a pure supporting electrolyte. Three differently treated GC electrodes were employed: (A-a) the GC electrode was polished with $0.3 \mu \mathrm{m}$ alumina powder, (A-b) the GC electrode was electrochemically oxidized in the $0.1 \mathrm{M} \mathrm{H}_{2} \mathrm{SO}_{4}$ solution, (A-c) and the electrochemically oxidized GC electrode was immersed in the gold nanoparticle solution. (B) Cyclic voltammograms in a pure supporting electrolyte. On the GC electrode, the gold nanoparticles were adsorbed, and a 2 aminoethanethiol self-assembled monolayer was further formed over the adsorbed gold nanoparticles. Scan rate: $100 \mathrm{mV} / \mathrm{s}$. Supporting electrolyte: $0.1 \mathrm{M}$ phosphate buffer (pH 6.0, Ar-purged).

monolayer of a thiol compound with a functional group such as an amino or carboxylic group can make a modified structure of the electrode surface. ${ }^{19,20}$ In the present study, the 2aminoethanethiol was chosen because it is soluble in an aqueous solution and its self-assembled monolayer on a gold electrode can provide sites with amino groups, which allow various chemical compounds to be attached. ${ }^{19,20}$ The GC electrode with the gold nanoparticles adsorbed as in Fig. 2A-c was further 
immersed in a $20 \mathrm{mM}$ 2-aminoethanethiol solution for $1 \mathrm{~h}$ to form its self-assembled monolayer. The electrode was then rinsed with deionized water and transferred to the $0.1 \mathrm{M}$ phosphate buffer to obtain the voltammograms in Fig. 2B. A broad cathodic wave at around $-0.75 \mathrm{~V}$ was measured in the first cathodic scanning. In the second scanning, the wave disappeared, giving a voltammogram similar to that of Fig. 2A-c. It was reported that various adsorbed thiol compounds were reductively desorbed from the gold surface. ${ }^{18,21,22}$ It is evident that the wave at $-0.75 \mathrm{~V}$ was due to the reductive desorption of the adsorbed 2-aminoethanethiol monolayer. A similar cathodic wave was also measured with the commonly used gold plate electrode after the 2-aminoethanethiol monolayer was adsorbed. Because the thiol compounds were desorbed from the surface during the negative potential scanning, no wave was observed in the second scanning with the bare nanoparticles. The presence of the thiol reduction wave confirms the presence of the gold nanoparticles on the electro-oxidized GC electrode, because the self-assembled monolayers were formed over the gold nanoparticle surfaces. After the wave disappeared, the electrode was immersed into the 2-aminoethanethiol solution again and the same cathodic wave at $-0.75 \mathrm{~V}$ was obtained, implying that the nanoparticles remain quite stable on the GC surface. When the gold nanoparticles on the electro-oxidized GC electrode were adsorbed, the immersion time in the gold colloidal solution was varied; with the prepared electrode, the thiol reduction wave was measured. In $40 \mathrm{~min}$ of the immersion time, the reduction wave at $-0.75 \mathrm{~V}$ reached a limited maximum size, which indicates that at least $40 \mathrm{~min}$ were required to obtain the equilibrated state in adsorbing the gold nanoparticles on the electrode surface.

Dependence of the amount of the gold nanoparticles on the degree of the electrochemical oxidation of the GC electrode

The relationship between the amounts of the adsorbed nanoparticles and the degree of the surface oxidation of the GC electrode was investigated as their adsorption was induced by the electrochemical oxidation treatment. Because the reduction of the adsorbed 2-aminoethanethiol appeared as a cathodic wave at $-0.75 \mathrm{~V}$ as in Fig. 2B, the charge under the wave is related to the surface coverage of the 2-aminoethanethiol monolayer on the adsorbed nanoparticles. The magnitude of the charge is then thought to be proportional to the amount of the adsorbed nanoparticles. The charge under the wave at $-0.75 \mathrm{~V}$ in Fig. $2 \mathrm{~B}$ was roughly estimated from the area, which was obtained by shifting the second scanned voltammogram in Fig. 2B up to the first scanned voltammogram and subtracting it from the first scanned voltammogram. The degree of the surface oxidation of the GC electrode was controlled by varying the applying time of the potential of $2.0 \mathrm{~V}$ in the oxidation pretreatment. The estimated charges are plotted against the oxidation time in Fig. 3. The amount of adsorbed nanoparticles increased with oxidation time and it reached a limiting value in about $6 \mathrm{~min}$. It is known that the electrochemical oxidation of the GC electrode forms micropores, giving a rough surface. ${ }^{14}$ In the previous studies, the penetration of catechol was limited at a finite depth of the micropores ${ }^{14}$ but copper ions reached into their deep insides. ${ }^{23}$ The result shown in Fig. 3 implies that the gold nanoparticles might not penetrate into the micropores, and might reside only on the outer surface probably because their sizes are larger than those of the micropores.

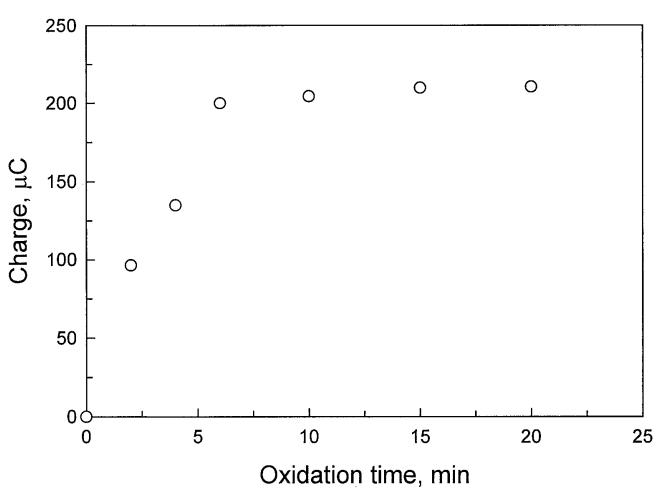

Fig. 3 Dependence of the amount of the gold nanoparticles on the degree of the electrode oxidation. On the GC electrode, the gold nanoparticles were first adsorbed and the 2-aminoethanethiol selfassembled monolayer was consequently formed. The charge for the reduction of the adsorbed 2-aminoethanethiol was estimated by subtracting the baseline from the cathodic wave at $-0.75 \mathrm{~V}$ in Fig. $2 \mathrm{~B}$, and plotted against the applying time for the electrochemical oxidation of the GC electrode.

\section{Conclusions}

The present study shows a new simple method for the adsorption of the gold nanoparticles on the GC electrode. They were evenly adsorbed on the electrochemically-pretreated electrode and remained quite stable during the electrochemical measurements. No adsorption occurred on the alumina-polished electrode. At present stage, we propose the origin of the interaction between the particles and the electrode surface to be electrostatic. The electrochemical oxidation of the GC electrode introduces various oxygen-containing functional groups on the surface, such as carboxylic acids, acid anhydrides, alcohols, phenols, and quinines. ${ }^{12}$ The surface becomes hydrophilic and several ionic compounds are adsorbed by the electrostatic interaction. ${ }^{14,23}$ The negatively charged particles ${ }^{24,25}$ may be attracted to the hydrophilic GC surface. Another property of the gold colloidal particles is their unstableness in the solution; they are easily aggregated together. ${ }^{24,25}$ This property may be also the driving power for the nanoparticles to be adsorbed on the surface.

On the adsorbed nanoparticles, the 2-aminoethanethiol selfassembled monolayer was formed and the reductive desorption wave was measured. It is expected that the amino group can provide sites for attachments of other chemical compounds. New surface structures over the adsorbed gold nanoparticles will be designed and used as catalysts in the electrode reactions for future studies. Further characterization studies need to be carried out to understand the origin of the interaction for the adsorption.

\section{Acknowledgements}

This work was supported by a Korea Research Foundation Grant (KRF-2002-070-C00066).

\section{References}

1. H. S. Nalwa, "Handbook of Nanostructured Materials and Nanotechnology", 2000, Academic Press, San Diego. 
2. A. C. Templeton, W. P. Wuelfing, and R. W. Murray, Acc. Chem. Res., 2000, 33, 27.

3. M. Haruta, Catal. Today, 1997, 36, 153.

4. P. Mukherjee, C. R. Patra, A. Ghosh, R. Kumar, and M. Sastry, Chem. Mater., 2002, 14, 1678.

5. I. Amlani, A. M. Rawlett, L. A. Nagahara, and R. K. Tsui, Appl. Phys. Lett., 2002, 80, 2761.

6. M. S. El-Deab and T. Ohsaka, Electrochem. Commun., 2002, 4, 288

7. M. M. Maye, Y. Lou, and C.-J. Zhong, Langmuir, 2000, 16,7520 .

8. J. A. Harnish, A. D. Pris, and M. D. Porter, J. Am. Chem. Soc., 2001, 123, 5829.

9. C. J. Zhong, W. X. Zheng, and F. L. Leibowitz, Electrochem. Commun., 1999, 1, 72.

10. F. L. Leibowitz, W. Zheng, M. M. Maye, and C.-J. Zhong, Anal. Chem., 1999, 71, 5076.

11. M. O. Finot, G. D. Braybrook, and M. T. McDermott, $J$. Electroanal. Chem., 1999, 466, 234.

12. R. L. McCreery, in "Electroanalytical Chemistry", ed. A. J. Bard, 1991, Vol. 17. Marcel Dekker, New York, 221.

13. G. W. Hance and T. Kuwana, Anal. Chem., 1987, 59, 131.

14. T. Nagaoka and T. Yoshino, Anal. Chem., 1986, 58, 1037.
15. T. Nagaoka, T. Fukunaga, T. Yoshino, I. Watanabe, T. Nakayama, and S. Okazaki, Anal. Chem., 1988, 60, 2766.

16. K. S. Mayya, V. Patil, and M. Sastry, Langmuir, 1997, 13, 2575.

17. G. E. Cabaniss, A. A. Diamantis, W. R. Murphy, Jr., R. W. Linton, and T. J. Meyer, J. Am. Chem. Soc., 1985, 107, 1845.

18. C. A. Widrig, C. Chung, and M. D. Porter, J. Electroanal. Chem., 1991, 310, 335.

19. M. Wirde, U. Gelius, and L. Nyholm, Langmuir, 1999, 15, 6370 .

20. A. Riklin and I. Willner, Anal. Chem., 1995, 67, 4118.

21. T. W. Schneider and D. A. Buttry, J. Am. Chem. Soc., 1993, 115, 12391.

22. J. J. Calvente, Z. Kovacova, M. D. Sanchez, R. Andreu, and W. R. Fawcett, Langmuir, 1996, 12, 5696.

23. H. Shin, M. Park, A. Kim, and C. Kang, J. Electroanal. Chem., 2003, 547, 143.

24. C. S. Weisbecker, M. V. Merritt, and G. M. Whitesides, Langmuir, 1996, 12, 3763.

25. K. S. Mayya, V. Patil, and M. Sastry, Langmuir, 1997, 13, 3944. 